\title{
Editorial Note for Journal of Clinical Infectious Diseases: Open Access
}

\author{
Aniruddha Ghosh* \\ Department of Pediatric Medicine, Institute of Child Health, Kolkata, West Bengal, India
}

\section{Editorial Note}

Journal of Clinical Infectious Diseases: Open Access is a Peer-reviewed medical journal that remembers a wide scope of fields for its control to make a platform for the authors to make their contributions towards the journal. The editorial office promises a thorough peer review of the submitted original manuscripts to ensure quality. This Scientific journal covers the accompanying points extensively such gastroenterologists, virologists, clinical pathologists, pulmonologists, clinical and lab professionals, academicians and researchers keen in investigating and introducing the clinical implications of infectious diseases, suggesting therapy and cure.

How New Technologies Could Help in Scrub Typhus, A Case of Successful Treatment of Septic Shock Secondary to Scrub Typhus; Azlina Ibrahim, et al. wrote a case report about Authors showed through this work, A 24-years of age Malaysian man introduced to the crisis office with a 9-day history of fever, migraine, diarrhea and loss of hunger. He denied hack or runny nose. Fourteen days before ailment, he went to National Park in Pahang, Malaysia for wilderness journeying.

Physical assessment uncovered nearness of two eschar estimated 7 $\mathrm{mm}$ beneath his correct axilla and left lower arm. Chest auscultation and stomach assessment were mediocre. Research center test uncovered no leukopenia, thrombocytopenia or pallor however raised serum Aspartate Aminotransferase (AST), Alanine Transferase (ALT) and Lactate Dehydrogenase.

Scrub typhus is a zoonosis brought about by Orientia tsutsugamushi, a gramnegative intracellular bacterium. Understanding regularly gave unexpected beginning of fever (typically 8 days to 16 days) following chomps from tainted parasite. Other vague manifestations incorporate myalgia, rashes and migraine which additionally found in instances of leptospirosis, dengue, and malaria. Acute respiratory distress syndrome is a notable inconvenience for clean typhus because of microangiopathic measure prompting central vasculitis show as bronchitis, interstitial pneumonitis or even pleural radiation.

\section{Conclusion}

Scrub typhus has showed up as one of the significant reason for intense febrile ailment which has been ignored for a long time. The determination of scour typhus can be trying due to the vague clinical indications and signs that can impersonate other zoonotic infection. Accordingly an exhaustive history and point by point physical assessment are imperative to help the determination of scour typhus. Clinicians must have a high record doubt of scour typhus particularly to those patients who dwell or travel to endemic zone. A deferral of treatment may bring about serious confusions and passing. Consistent training project and consciousness of the infection as one of the differential finding of intense febrile sickness in locale where the ailment are endemic must be pushed.

\section{Declaration of Patient Consent}

The authors ensure that they have gotten all fitting patient assent structures. The patients comprehend that their names and initials won't be distributed and due endeavors will be made to hide their personality, however obscurity can't be ensured.

The focal point of the high impact factor journal is the disease process clarification and association techniques guaranteeing upgraded results for the patient. Persistent point of view fulfillment, healthy life, quality treatment, correspondence, and wellbeing proficiency and their role in growing new health care services programs and clinical results are significant zones of enthusiasm for this journal.

The journal is using online manuscript submission, review and tracking system. The review process is performed by the Editorial board members from the journal of general practice or outside specialists; at least two reviewer's approval trailed by the editor is required for the acknowledgment of any citable citable manuscript.

*Address for Correspondence: Aniruddha Ghosh, Department of Pediatric Medicine, Institute of Child Health, Kolkata, West Bengal, India, E-mail: aniruddha179@gmail.com

Copyright: @ 2020 Aniruddha Ghosh. This is an open-access article distributed under the terms of the Creative Commons Attribution License, which permits unrestricted use, distribution, and reproduction in any medium, provided the original author and source are credited. 